CENTRE FOR EMEA BANKING, FINANCE \& ECONOMICS

The Pricing of Contingent Claims in a Multivariate Gamma Distributed Economy

Luiz Vitiello

Ivonia Rebelo

Working Paper Series

No 21/11 


\title{
The Pricing of Contingent Claims in a Multivariate Gamma Distributed Economy
}

\author{
Luiz Vitiello* and Ivonia Rebelo
}

${ }^{*}$ Luiz Vitiello is the corresponding author. Both authors are at London Metropolitan Business School, London Metropolitan University, UK. Please send all correspondence to London Metropolitan Business School, 84 Moorgate, London EC2M 6SQ, UK. Tel: +44 (0) 207320 1514. Luiz Vitiello is a Senior Lecturer in Finance, email: l.vitiello@londonmet.ac.uk. Ivonia Rebelo is a Principal Lecturer in Economics, email: i.rebelo@londonmet.ac.uk. We would like to thank Keith Cuthbertson and Ser-Huang Poon for valuable comments. 


\title{
The Pricing of Contingent Claims in a Multivariate Gamma Distributed Economy
}

\begin{abstract}
In this paper we establish a Risk Neutral Valuation Relationship and develop a framework for pricing multivariate European-style contingent claims in a discrete-time economy using a multivariate gamma distribution. In our framework, risk neutrality is obtained by using market equilibrium conditions, leading to preference-free contingent claim pricing equations. Multivariate contingent claim pricing models are of particular interest when payoffs depend on two or more stochastic variables, such as options to exchange one asset for another, options on mutual funds, and options with a stochastic strike price in general. In our model each underlying stochastic variable depends on a systematic gamma distributed term and on an idiosyncratic one, where the former has a direct impact on the correlation structure of the underlying variables. To illustrate the applicability of our framework, we present multivariate gamma distributed versions of well-known multivariate normally/lognormally distributed contingent claim pricing formulae. The gamma distribution is particularly suitable to price stochastic variables that present implied volatilities that are an increasing function of the strike price.

Keywords: Multivariate Gamma Distribution, Risk Neutral Valuation Relationship, Multivariate Contingent Claim, Stochastic Strike Price, General Equilibrium.
\end{abstract}




\section{The Pricing of Contingent Claims in a Multivariate Gamma Distributed Economy}

\section{Introduction}

In this paper we establish a Risk Neutral Valuation Relationship (RNVR) and develop a framework for pricing multivariate European-style contingent claims in a discrete-time economy using a multivariate gamma distribution. Risk neutrality is obtained by using market equilibrium conditions, which leads to preference-free contingent claim pricing equations. The framework developed here can be useful for pricing contingent claims whose payoffs depend on more than one underlying stochastic variables, such as options to exchange one asset for another, options on mutual funds, currency option bonds, and options with a stochastic strike price in general.

Most of the previous studies on the pricing of multivariate contingent claims rely, in some way, on the assumption of normal or lognormal random variables i.e. extensions of the Black and Scholes (1973) model (see for instance Margrabe (1978), Stulz (1982), Stapleton and Subrahmanyam (1984), Johnson (1987), Camara (2005)). In this paper we depart from the well known Gaussian framework by letting the underlying stochastic variables have a multivariate gamma distribution. In our model the distribution of each underlying stochastic variables depends on two independent gamma distributed terms, where one of them can be regarded as idiosyncratic and the other one as systematic. While the idiosyncratic terms are, of course, independent of each other, the systematic term has a direct impact on all 
underlying stochastic variables and on their correlation structure. As our framework is developed in a multivariate setting, it extends previous contingent claim pricing models which are based on only one gamma distributed underlying stochastic variable (see for instance Heston (1993), Schroder (2004), Vitiello and Poon (2010)).

The gamma distribution contains the normal distribution as a limiting case and, as the lognormal distribution, it lies on a single line in the skewnesskurtosis plane (see Johnson, Kotz and Balakrishnan (1994)). While the implied volatility obtained from the lognormal distributed Black and Scholes (1973) model is parallel to the $x$ axis, the implied volatility obtained from the gamma distribution is an increasing function of the strike price, which makes it particularly interesting for pricing agricultural commodities for instance (Zhou (1988), Savickas (2005)). Also, the gamma distribution has been widely applied to model natural events, in particular rainfall (see for example Simpson (1972), Griffiths (1990)).

In order to illustrate the applicability of our framework, we present preference free option pricing models that depend on one or more underlying stochastic variables. Specifically, we present multivariate gamma distributed versions of well-known multivariate normally/lognormally distributed contingent claim pricing formulae. In this case, the limits of integration of the pricing equation may involve functions of the underlying stochastic variables themselves, which increases the complexity of the pricing equation. In such situations, numerical solutions can be easily obtained.

This article is organised as follows: in Section 2 the basic economy and the multivariate gamma distribution are introduced and the forward asset 
specific pricing kernel is obtained. In Section 3 an equilibrium relationship for the price of the underlying stochastic variables is obtained and a general multivariate gamma contingent claim pricing model is derived. Special cases of the general pricing model are introduced and new preference free option pricing models are presented in Section 4. In Section 5 the estimation of parameters is briefly discussed. Section 6 concludes. Proofs and a list of relevant variables are provided in the Appendix.

\section{The Valuation Model}

\subsection{The Economy}

The basic setting introduced in this section is similar to the economy developed by Stapleton and Subrahmanyam (1984) and Camara (2005), in which there is a representative investor who maximises her expected utility of terminal consumption, $\operatorname{Max} E[U]$, where $U$ is the investor's utility function of consumption and $E[\cdot]$ is the expectation taken with respect to the physical probability measure.

In equilibrium, it follows from the first order condition for a maximum that

$$
\begin{aligned}
\mathbf{P}(\mathbf{V}(\mathbf{X})) & =\frac{E\left[\mathbf{V}(\mathbf{X}) U_{C}\right]}{E\left[U_{C}\right]} \\
& =E[\mathbf{V}(\mathbf{X}) \phi(\mathbf{X})],
\end{aligned}
$$

where $U_{C}$ is the first derivative of the utility function with respect to consumption, $\mathbf{V}(\mathbf{X})$ is the vector of payoffs of the claims $\mathbf{V}(\cdot)$ as a function of the payoff of underlying stochastic variables $\mathbf{X}, \mathbf{P}(\mathbf{V}(\mathbf{X}))$ is the vector of 
current forward prices of $\mathbf{V}(\mathbf{X})$ and

$$
\phi(\mathbf{X})=\frac{E\left[U_{C} \mid \mathbf{X}\right]}{E\left[U_{C}\right]}
$$

is defined as the forward asset-specific pricing kernel (see Brennan, 1979).

Equation (2.1) is referred to as the basic valuation equation. It follows that the forward value of the underlying random variables can be obtained from equation (2.1). For instance, if $\mathbf{V}(\mathbf{X})=\mathbf{X}$ then $\mathbf{P}(\mathbf{X})=$ $E[\mathbf{X} \phi(\mathbf{X})]$, where $\mathbf{P}(\mathbf{X})$ is the vector of forward values of the underlying random variables $\mathbf{X}$.

\subsection{The Multivariate Gamma Distribution}

There are several ways of constructing a multivariate gamma distribution. An overview is given in Kotz, Balakrishnan and Johnson (2000). Here we follow the Mathai and Moschopoulos (1991) approach, which amongst other features, guarantees gamma distributed marginal densities.

Definition 2.1 (The gamma distribution) The random variable $y$ is said to be gamma distributed, $y \sim G(p, \sigma, \mu)$, when its three-parameter density function is given by

$$
f(y ; p, \sigma, \mu)=\frac{(y-\mu)^{p-1}}{\sigma^{p} \Gamma(p)} \exp \left[-\left(\frac{y-\mu}{\sigma}\right)\right], \quad \mu<y<\infty,
$$

where $p>0$ is the shape parameter, $\sigma>0$ is the scale parameter, $\mu$ is the location parameter and $\Gamma(\cdot)$ is the gamma function.

Proposition 2.2 (The multivariate gamma distribution) If

$$
z_{i}=\frac{\sigma_{i}}{\sigma_{0}} y_{0}+y_{i}, \quad i=1, \ldots, M
$$


then the vector $\mathbf{Z}=\left(z_{1}, \ldots, z_{M}\right)$ is said to have a multivariate gamma distribution, where $y_{i} \sim G\left(p_{i}, \sigma_{i}, \mu_{i}\right)$, for $i=0, \ldots, M$, are mutually independent. Thus, $z_{i} \sim G\left(p_{0}+p_{i}, \sigma_{i}, \mu_{i}+\mu_{0} \frac{\sigma_{i}}{\sigma_{0}}\right)$.

Given Proposition 2.2, one can think of $y_{i}$ as an idiosyncratic gamma distributed term and $y_{0}$ as a gamma distributed systematic term (a disturbance on $y_{i}$ ), leading to correlated $z_{i}^{\prime} s$. Note that according to Proposition 2.2 the $y^{\prime} s$ are mutually independent but the $z^{\prime} s$ are positively correlated with $\operatorname{Cov}\left(z_{i}, z_{j}\right)=p_{0} \sigma_{i} \sigma_{j}, i \neq j$.

Also, considering Proposition 2.2, the joint density of $z_{1}, \ldots, z_{M}$ is given by

$$
\begin{aligned}
f\left(z_{1}, \ldots, z_{M}\right)= & \prod_{i=1}^{M} \int_{y_{0}} \frac{\left(y_{0}-\mu_{0}\right)^{p_{0}-1}}{\Gamma\left(p_{0}\right) \Gamma\left(p_{i}\right) \sigma_{0}^{p_{0}} \sigma_{i}^{p_{i}}}\left(z_{i}-y_{0} \sigma_{i} / \sigma_{0}-\mu_{i}\right)^{p_{i}-1} \\
& \exp \left(-\left(y_{0}-\mu_{0}\right) / \sigma_{0}-\left(z_{i}-y_{0} \sigma_{i} / \sigma_{0}-\mu_{i}\right) / \sigma_{i}\right) d y(2.5)
\end{aligned}
$$

which is obtained by using equation (2.3), Proposition 2.2 and integrating with respect to $y_{0}$.

\subsection{The Asset Specific Pricing Kernel}

In this subsection we present three propositions. The first one states that if the $m^{\text {th }}$ element of $\mathbf{Z}$ represents consumption, then $C=\exp \left(z_{M}\right)$ and $x_{i}=\exp \left(z_{i}\right)$ for $i=1, \ldots, M-1$. The second states that investors have a power marginal utility function, which denotes Constant Proportional Risk Aversion. These results and Proposition 2.2 lead to the functional form for the asset specific pricing kernel, presented in the third proposition, which is used in the subsequent sections to price the underlying stochastic variables $x_{1}, x_{2}, \ldots, x_{M-1}$, and contingent claims written on them. 
Proposition 2.3 (The distribution of the underlying stochastic variables) The natural logarithm of the terminal consumption and the natural logarithm of the payoff of the underlying stochastic variables have a multivariate gamma as in Proposition (2.2).

Proposition 2.4 (The marginal utility function) The representative investor has a marginal utility function given by $U_{C}=C^{\gamma}$, where the constant $\gamma$ is a preference parameter.

Proposition 2.5 (The forward asset specific pricing kernel) Assume that propositions 2.2, 2.3 and 2.4 hold. Then for $\mathbf{X}=\left(x_{1}, \ldots, x_{M-1}\right)$ the forward asset specific pricing kernel is given by

$$
\begin{aligned}
\phi(\mathbf{X})= & \prod_{i=1}^{M-1} \frac{\sigma_{i}^{p_{0}} \Gamma\left(p_{0}+p_{i}\right)}{\sigma_{0}^{p_{0}} \Gamma\left(p_{0}\right) \Gamma\left(p_{i}\right)} \frac{\left(1-\gamma \sigma_{M}\right)^{p_{0}}}{\left(\ln x_{i}-\mu_{i}^{*}\right)^{p_{0}+p_{i}-1}} \\
& \int_{x_{0}} x_{0}^{p_{0}-1}\left(\ln x_{i}-\frac{\sigma_{i}}{\sigma_{0}}\left(x_{0}+\mu_{0}\right)-\mu_{i}\right)^{p_{i}-1} \exp \left(x_{0} \gamma \frac{\sigma_{M}}{\sigma_{0}}\right) d x(2.6)
\end{aligned}
$$

where $\mu_{i}^{*}=\mu_{0} \sigma_{i} / \sigma_{0}+\mu_{i}$

Proof. See Appendix B.

\section{Prices in Equilibrium}

Using equation (2.1), propositions 2.3 and 2.5, and letting $V\left(x_{i}\right)=x_{i}$ for $i=1,2, \ldots M-1$ the equilibrium relationship for the forward price of the 
underlying stochastic variables is given by

$$
\begin{aligned}
P\left(x_{i}\right) & =E\left[x_{i} \phi(\mathbf{X})\right] \\
& =E\left[\exp \left(z_{i}\right) \phi(\mathbf{X})\right] \\
& =\int_{z_{1}} \int_{z_{2}} \cdots \int_{z_{M-1}} \exp \left(z_{i}\right) \phi(\mathbf{X}) f\left(z_{1}, z_{2}, \ldots, z_{M-1}\right) d z_{1} d z_{2} \ldots d z_{M-1} \\
& =\frac{\left(1-\gamma \sigma_{M}\right)^{p_{0}}}{\left(1-\sigma_{i}-\gamma \sigma_{M}\right)^{p_{0}}} \frac{\exp \left(\mu_{i}^{*}\right)}{\left(1-\sigma_{i}\right)^{p_{i}}} \quad \text { for } i=1,2, \ldots M-1
\end{aligned}
$$

where $\mathbf{P}(\mathbf{X})=\left(P\left(x_{1}\right), \ldots, P\left(x_{M-1}\right)\right)$.

It is interesting to note that, as in the univariate gamma models of Heston (1993) and Vitiello and Poon (2010), the preference parameter has a direct impact on the scale parameter. This contrasts, for instance, with the multivariate (transformed) normal economies of Stapleton and Subrahmanyam (1984) and Camara (2005), in which the preference parameter impacts on the location parameter only.

In the particular case of multivariate gamma distributed payoffs, it is possible to use the forward equilibrium relationship in equation (3.1) to solve for the preference parameter $\gamma$, yielding the following relationship

$$
\gamma=\left[1-\frac{\sigma_{i}\left(1-\sigma_{i}\right)^{p_{i} / p_{0}} \exp \left(-\mu_{i}^{*} / p_{0}\right)}{\left(1-\sigma_{i}\right)^{p_{i} / p_{0}} \exp \left(-\mu_{i}^{*} / p_{0}\right)-P\left(x_{i}\right)^{-1 / p_{0}}}\right] \frac{1}{\sigma_{M}} \forall i=1,2 \ldots M-1
$$

The equation above is of particular interest since it shows that the preference parameter can be expressed as a function of the price of the underlying stochastic variables. This allows us to obtain the forward price of contingent claims in a preference free form such that $\mathbf{P}(\mathbf{V}(\mathbf{X}))=E[\mathbf{V}(\mathbf{X}) \phi(\mathbf{X})]=$ $E^{Q}[\mathbf{V}(\mathbf{X})]$.

Equations (3.1) and (3.2) prove the following proposition and corollary. 
Proposition 3.1 (The existence of a RNVR) If the payoff of the stochastic variables have a distribution according to Proposition 2.3 and the asset specific pricing kernel in Proposition 2.5 holds then a risk neutral valuation relationship exists.

Corollary 3.2 (Sufficient condition for a RNVR) A sufficient condition for a RNVR to hold under Proposition 2.3 is that the marginal utility function of consumption of the representative investor has a power form as in Proposition 2.4.

According to Proposition 3.1, in a multivariate gamma economy risk neutrality is achieved by construction, based on market equilibrium conditions alone. Thus, it is possible to price more complex payoff functions. Using Proposition 3.1 and following the same procedure adopted in equation (3.1), one obtains

$$
\begin{aligned}
\mathbf{P}(\mathbf{V}(\mathbf{X}))= & E[\mathbf{V}(\mathbf{X}) \phi(\mathbf{X})] \\
= & E\left[V\left(\exp \left(z_{1}, \ldots, z_{M-1}\right)\right) \phi(\mathbf{X})\right] \\
= & \int_{z_{1}} \int_{z_{2}} \cdots \int_{z_{M-1}} V\left(\exp \left(z_{1}, \ldots, z_{M-1}\right)\right) \phi(\mathbf{X}) \\
& f\left(z_{1}, z_{2}, \ldots, z_{M-1}\right) d z_{1} d z_{2} \ldots d z_{M-1} .
\end{aligned}
$$

Specific forms of equation (3.3) are discussed in the following section. 


\section{Applications}

\subsection{The Vanilla Call Option}

We are interested in a call option written on $x_{i}$ with strike price $K$, and payoff $V\left(x_{i}\right)=\operatorname{Max}\left(x_{i}-K, 0\right)$. Using Proposition 2.3, the price of such option can be obtained by the following specific forms of equation (3.3)

$$
\begin{aligned}
P\left(V\left(x_{i}\right)\right) & =E\left[V\left(x_{i}\right) \phi\left(x_{i}\right)\right] \\
& =\int_{z_{i}} \operatorname{Max}\left(\exp \left(z_{i}\right)-K, 0\right) \phi\left(x_{i}\right) f\left(z_{i}\right) d z_{i} .
\end{aligned}
$$

Using propositions 2.2 and 2.5 one obtains

$$
\begin{aligned}
P\left(V\left(x_{i}\right)\right)= & \int_{z_{i}} \operatorname{Max}\left(\exp \left(z_{i}\right)-K, 0\right) \frac{\left(1-\gamma \sigma_{M}\right)^{p_{0}}}{\sigma_{0}^{p_{0}} \sigma_{i}^{p_{i}} \Gamma\left(p_{0}\right) \Gamma\left(p_{i}\right)} \exp \left(-\frac{z_{i}-\mu_{i}^{*}}{\sigma_{i}}\right) \\
& \int_{x_{0}} x_{0}^{p_{0}-1}\left(z_{i}-x_{0} \sigma_{i} / \sigma_{0}-\mu_{i}^{*}\right)^{p_{i}-1} \exp \left(x_{0} \gamma \sigma_{M} / \sigma_{0}\right) d x_{0} d z_{i},
\end{aligned}
$$

which after changing variables, interchanging integrals, using the equilibrium and simplifying yields relationship in equation (3.2) and simplifying yields the multivariate gamma vanilla call option

$$
\begin{aligned}
P\left(V\left(x_{i}\right)\right)= & \int_{0}^{1} B\left(p_{0}, p_{i}\right)^{-1} w_{0}^{p_{0}-1}\left(1-w_{0}\right)^{p_{i}-1}\left(\theta_{i} / F_{i}\right)^{p_{0}} \\
& {\left[\exp \left(\mu_{i}^{*}\right)\left(1-\left(1-\theta_{i} / F_{i}\right) w_{0}-\sigma_{i}\right)^{-\left(p_{0}+p_{i}\right)}\left[1-G\left(d_{1}\right)\right]\right.} \\
& \left.-K\left(1-\left(1-\theta_{i} / F_{i}\right) w_{0}\right)^{-\left(p_{0}+p_{i}\right)}\left[1-G\left(d_{2}\right)\right]\right] d w_{0},
\end{aligned}
$$

where $B\left(p_{0}, p_{i}\right)=\Gamma\left(p_{0}\right) \Gamma\left(p_{i}\right) / \Gamma\left(p_{0}+p_{i}\right)$ is the beta function,

$$
\begin{aligned}
\sigma_{i}^{*} & =\left(1-\sigma_{i}\right)^{p_{i} / p_{0}} \\
\mu_{i}^{*} & =\mu_{0} \sigma_{i} / \sigma_{0}+\mu_{i}
\end{aligned}
$$




$$
\begin{gathered}
\theta_{i}=\sigma_{i} \sigma_{i}^{*} \exp \left(-\mu_{i}^{*} / p_{0}\right) \\
F_{i}=\sigma_{i}^{*} \exp \left(-\mu_{i}^{*} / p_{0}\right)-P\left(x_{i}\right)^{-1 / p_{0}} \\
d_{1}=\left(\ln (K)-\mu_{i}^{*}\right)\left(1-\left(1-\theta_{i} / F_{i}\right) w_{0}-\sigma_{i}\right) / \sigma_{i} \\
d_{2}=d_{1}+\left(\ln (K)-\mu_{i}^{*}\right)
\end{gathered}
$$

for $\sigma_{i}<1, \sigma_{i}^{*}>0, F_{i}>0, \ln (K)>\mu_{i}^{*}$.

We calculated theoretical prices according to equation (4.2) and then searched for a value for the Black and Scholes (1973) volatility parameter that would make the Black and Scholes price equal to the theoretical price for a range of strike prices. These (implied) volatilities are an increasing function of the strike price, as shown in Figure 1, which depicts the implied volatility for different strikes prices, with $\mu_{0}=\mu_{2}=1, \sigma_{0}=\sigma_{1}=\sigma_{2}=0.5, p_{0}=1$; the solid line represents $p_{2}=2$ and the dotted line $p_{2}=1$. In particular, increasing (decreasing) $\sigma_{0}$ leads to a higher (smaller) implied volatility while increasing (decreasing) all other parameters leads to an opposite result.

[Figure 1 about here]

\subsection{An Option on a Mutual Fund}

The option pricing formula in equation (4.2) can be easily extended to price other types of derivatives, such as an option written on a mutual fund. Following Stapleton and Subrahmanyam (1984), the value of a company can be viewed as a vanilla call option given by $s_{i}=\max \left(x_{i}-y_{i}, 0\right)$, where $s_{i}$ is the equity in the $i^{t h}$ levered company for $i=1, \ldots, M-1, x_{i}$ is the multivariate gamma distributed market value of the $i^{\text {th }}$ company, and $y_{i}$ is company $i$ 
promised payment to bondholders. Clearly, if $x_{i}-y_{i}<0$ shareholders will default on the debt.

Since a mutual fund can be viewed as a weighted average of such companies, its value is given by $H=\sum_{i} \alpha_{i} s_{i}=\sum_{i} \alpha_{i} \max \left(x_{i}-y_{i}, 0\right)$, where $\alpha_{i}$, $\sum_{i} \alpha_{i}=1$, is the weight of security $i$ in the mutual fund. Thus the value of a call option with strike price $K$ written on this mutual fund can be obtained by the following equation

$$
H=\max \left(\sum_{i=1}^{M-1} \alpha_{i} \max \left(x_{i}-y_{i}, 0\right)-K, 0\right) .
$$

Considering the similarity between $s_{i}$ and the payoff function of a call option $V\left(x_{i}\right)=\operatorname{Max}\left(x_{i}-K, 0\right)$, each one of the $M-1$ levered companies can be priced as in Example 4.1, yielding the following general solution

$$
H=\prod_{i=1}^{M-1} \int_{y_{i}} \max \left[\sum_{i=1}^{M-1} \alpha_{i}\left(x_{i}-y_{i}\right)-K, 0\right] \phi(\mathbf{X}) f\left(x_{1}, x_{2}, \ldots, x_{M-1}\right) d x_{i}
$$

which can be solved in a similar manner as in Section 4.1 by using equations (2.6) and (3.1).

\subsection{The Option to Exchange one Underlying Asset for Another}

The value of an option to exchange one underlying stochastic variable for another based on the Black and Scholes (1973) equation was first proposed by Margrabe (1978). Here we present, for a multivariate gamma distributed framework, an exact solution for a call option to exchange the stochastic random variable $x_{1}$ for $x_{2}$ with payoff function given by $V\left(x_{1}, x_{2}\right)=$ 
$\operatorname{Max}\left(x_{1}-x_{2}, 0\right)$. Using a specialization of equation (3.3) yields

$$
\begin{aligned}
P\left(V\left(x_{1}, x_{2}\right)\right) & =E\left[V\left(x_{1}, x_{2}\right) \phi\left(x_{1}, x_{2}\right)\right] \\
& =\int_{z_{1}} \int_{z_{2}} \operatorname{Max}(h(x), 0) \phi\left(x_{1}, x_{2}\right) f\left(z_{1}, z_{2}\right) d z_{1} d z_{2},
\end{aligned}
$$

where $h(x)=\exp \left(z_{1}\right)-\exp \left(z_{2}\right)$.

Using propositions 2.2 and 2.5, the definition of the beta function and simplifying it, one obtains

$$
\begin{aligned}
P\left(V\left(x_{1}, x_{2}\right)\right)= & \prod_{i=1}^{2} \int_{z_{1}} \int_{z_{2}} \operatorname{Max}(h(x), 0) \frac{\left(1-\gamma \sigma_{M}\right)^{p_{0}}}{\Gamma\left(p_{0}\right) \Gamma\left(p_{i}\right) \sigma_{i}^{p_{i}+p_{0}}} \\
& \exp \left(-z_{i} / \sigma_{i}+\mu_{i}^{*} / \sigma_{i}\right)\left(z_{i}-\mu_{i}^{*}\right)^{p_{0}+p_{i}-1} \int_{w_{0}} w_{0}^{p_{0}-1} \\
& \left(1-w_{0}\right)^{p_{i}-1} \exp \left(w_{0}\left(z_{i}-\mu_{i}^{*}\right) \gamma \sigma_{M} / \sigma_{i}\right) d w_{0} d z_{1} d z_{2} .
\end{aligned}
$$

After changing variables, interchanging integrals, using the equilibrium relationship in equation (3.2), the definition of the beta function yields and simplifying yields the option pricing equation to exchange one underlying asset for another

$$
\begin{aligned}
P\left(V\left(x_{1}, x_{2}\right)\right)= & \prod_{i=1}^{2} \int_{w_{0}} w_{0}^{p_{0}-1}\left(1-w_{0}\right)^{p_{i}-1} \frac{\left(\theta_{i} / F_{i}\right)^{p_{0}}}{\left(1-w_{0}\left(1-\theta_{i} / F_{i}\right)\right)^{p_{0}+p_{i}}} \\
& {\left[\frac{B\left(p_{0}, p_{1}\right)^{-1}}{\Gamma\left(p_{2}\right)} \frac{\exp \left(\mu_{1}^{*}\right)\left(1-w_{0}\left(1-\theta_{i} / F_{i}\right)\right)^{p_{0}+p_{1}}}{\left(1-\sigma_{1}-w_{0}\left(1-\theta_{i} / F_{i}\right)\right)^{p_{0}+p_{1}}}\right.} \\
& \int_{x_{2}} \exp \left(-x_{2}\right) x_{2}^{p_{0}+p_{2}-1}\left[1-G\left(d_{1}\right)\right] d x_{2} \\
& -\frac{B\left(p_{0}, p_{1}\right)^{-1}}{\Gamma\left(p_{2}\right)} \frac{\exp \left(\mu_{2}^{*}\right)\left(1-w_{0}\left(1-\theta_{i} / F_{i}\right)\right)^{p_{0}+p_{2}}}{\left(1-\sigma_{2}-w_{0}\left(1-\theta_{i} / F_{i}\right)\right)^{p_{0}+p_{2}}} \\
& \left.\int_{x_{2}} \exp \left(-x_{2}\right) x_{2}^{p_{0}+p_{2}-1}\left[1-G\left(d_{2}\right)\right] d x_{2}\right] d w_{0},
\end{aligned}
$$

where for $i=1,2, B\left(p_{0}, p_{i}\right)=\Gamma\left(p_{0}\right) \Gamma\left(p_{i}\right) / \Gamma\left(p_{0}+p_{i}\right)$ is the beta function,

$$
\sigma_{i}^{*}=\left(1-\sigma_{i}\right)^{p_{i} / p_{0}}
$$




$$
\begin{gathered}
\mu_{i}^{*}=\mu_{0} \sigma_{i} / \sigma_{0}+\mu_{i} \\
\theta_{i}=\sigma_{i} \sigma_{i}^{*} \exp \left(-\mu_{i}^{*} / p_{0}\right) \\
F_{i}=\sigma_{i}^{*} \exp \left(-\mu_{i}^{*} / p_{0}\right)-P\left(x_{i}\right)^{-1 / p_{0}} \\
d_{1}=\left(\frac{\sigma_{2} x_{2}}{1-w_{0}\left(1-\theta_{i} / F_{i}\right)}+\mu_{2}^{*}-\mu_{1}^{*}\right) \frac{1-\sigma_{1}-w_{0}\left(1-\theta_{i} / F_{i}\right)}{\sigma_{1}} \\
d_{2}=\left(\frac{\sigma_{2} x_{2}}{1-\sigma_{2}-w_{0}\left(1-\theta_{i} / F_{i}\right)}+\mu_{2}^{*}-\mu_{1}^{*}\right) \frac{1-w_{0}\left(1-\theta_{i} / F_{i}\right)}{\sigma_{1}}
\end{gathered}
$$

\section{$5 \quad$ Estimation of Parameters}

The applicability of equations (4.2), (4.3), and (4.5) depends on the estimation of the relevant parameters. One way of estimating the parameters is through historical data. If information on the idiosyncratic gamma distributed term is available, $y_{i}$ for $i=1, \ldots, M-1$, the method of moments can applied. Otherwise, if only information on $z_{i}$ is available, for $i=1, \ldots, M-1$, one can apply the methodology suggested by Mathai and Moschopoulos (1991) for instance.

However, parameters estimated through these methods capture events that have already passed and therefore may not be an appropriate estimate of their future behaviour. An alternative method is the use of parameters implied by the option pricing equation, as these are essentially forward looking (see Poon and Granger 2003). This method consists of using market option prices as an input to the model and then (numerically) searching for a value of the unknown parameters that minimise a certain objective function, such as the absolute (or squared) value of the difference between the current and 
the theoretical option prices based on the estimated parameters (see Mayhew 1995).

The estimation of unknown parameters using the implied method requires that the number of market option prices must be equal to or greater than the number of parameters to be estimated. Also, it may be necessary to impose arbitrary constraints on the value of the parameters in order to obtain a unique solution or to guarantee convergence. A potential source of error in the estimation comes from the fact that option prices are usually asynchronous, as trading does not necessarily takes place on the underlying asset and on all options at the same time. This may cause distortions on the estimation procedure, mainly with less liquid options.

\section{Conclusion}

In this paper we obtain a risk neutral valuation relationship for an economy in which the underlying stochastic variables are multivariate gamma distributed. This setting allows us to develop a framework for the pricing of contingent claims in a multivariate gamma distributed economy, hence extending the work of Stapleton and Subrahmanyam (1984) and Camara (2005) to a non-Gaussian setting. Risk neutrality is obtained by using market equilibrium conditions alone and the resulting contingent claim pricing equations are consequently free of preference parameters.

The gamma distribution may be particularly useful for pricing agricultural commodities, as it captures the increasing implied volatility of contin-

gent claims as a function of the strike price (see Zhou (1998), Vitiello and 
Poon (2010)). Thus one could price an option on a basket of agricultural commodities using the framework developed here, which also allows the impact of a systematic variable on option prices to be considered. In the particular case of agricultural commodities it could be temperature or rainfall, measured by the HDD/CDD (Heating Degree Day and Cooling Degree Days respectively) index futures or the Rainfall index futures for instance. Given the discussion in Section 5, the option formulae presented here can be applied even if such a systematic variable is not known. The only requirement is that there are more traded options than the number of unknown parameters.

The gamma distribution has been widely applied to model natural events, rainfall in particular. Thus, as suggested by Mathai and Moschopoulos (1991), the multivariate gamma distribution can be applied to check the impact that rain has on two uncorrelated streams. Considering the case in which these two uncorrelated streams pass through a town, rainfall may increase the chance of flooding. The framework developed here could help the pricing of contingent claims on flooding, for instance. It is important to note that in an incomplete market setting, such as the one related to flooding, prices obtained through the forward asset specific pricing kernel in equation (2.6) are not unique. However, actuaries have been using complete market techniques, such as the Esscher transform and Wang transform, to calculate premiums in an incomplete market (see Buhlmann (1980), Gerber and Shiu (1994), Wang (2002, 2003), for instance). If one accepts such constraints, the framework developed here could be used to price such payoffs. 


\section{Appendix A}

In this appendix, for convenience, we summarise the notation used in the text.

$M$ number of stochastic variables in the economy

$\mathbf{X}=\left(x_{0}, \ldots, x_{M}\right)$ vector of underlying stochastic variables

$V(\mathbf{X})$ payoff function of a claim as a function of $X$

$\mathbf{P}(\mathbf{V}(\mathbf{X}))$ vector of forward prices of $\mathbf{V}(\mathbf{X})$

$C$ Consumption

$U$ utility function of consumption

$U_{C}$ marginal utility function of consumption

$\gamma$ preference parameter

$\phi(\mathbf{X})$ forward asset specific pricing kernel

$p, \sigma, \mu$ distributional parameters

$f(z ; p, \sigma, \mu)$ marginal density function of $z$, with parameters $p, \sigma, \mu$

$f\left(z_{1}\right)$ marginal density function of $z_{1}$ (the notation is a short version of the one above)

$f\left(z_{1}, z_{2}\right)$ joint density of $z_{1}$ and $z_{2}$

$f\left(z_{1} \mid z_{2}\right)$ marginal density function of $z_{1}$ conditional to $z_{2}$.

$\mathbf{Z}=\left(z_{1}, \ldots, z_{M}\right)$ multivariate gamma vector

$z_{j}=y_{0} \sigma_{j} / \sigma_{0}+y_{j}$ for $j=1, \ldots M$

$x_{i}=\exp \left(z_{i}\right)$ for $i=1, \ldots M-1$

$C=\exp \left(z_{M}\right)$

$E(\cdot)$ expected value under the physical distribution

$E^{Q}(\cdot)$ expected value under the risk neutral distribution 


\section{Appendix B}

Proof. (Proof of Proposition 2.5) For ease of reading, this proof is divided in three parts, in which we obtain $E\left[U_{C}\right], E\left[U_{C} \mid x_{i}\right]$, and $\phi(\mathbf{X})$ respectively.

1. (The $E\left[U_{C}\right]$ ) From propositions $2.2,2.3$, and 2.4 we obtain

$$
\begin{aligned}
E\left[U_{C}\right]= & E\left(\exp \left(\gamma z_{M}\right)\right) \\
= & \int_{y_{0}} \int_{z_{M}} \frac{\exp \left(\gamma z_{M}\right)}{\Gamma\left(p_{0}\right) \sigma_{0}^{p_{0}} \Gamma\left(p_{M}\right) \sigma_{M}^{p_{M}}}\left(y_{0}-\mu_{0}\right)^{p_{0}-1} e^{-\left(y_{0}-\mu_{0}\right) / \sigma_{0}} \\
& \left(z_{M}-y_{0} \sigma_{M} / \sigma_{0}-\mu_{M}\right)^{p_{M}-1} e^{-\left(z_{M}-y_{0} \sigma_{M} / \sigma_{0}-\mu_{M}\right) / \sigma_{M}} d z_{M} d y_{0}
\end{aligned}
$$

which yields

$$
E\left(\exp \left(\gamma z_{M}\right)\right)=\frac{\exp \left(\gamma\left(\mu_{0} \sigma_{M} / \sigma_{0}+\mu_{M}\right)\right)}{\left(1-\gamma \sigma_{M}\right)^{p_{M}+p_{0}}}
$$

2. (The $E\left[U_{C} \mid x_{i}\right]$ ) Using propositions 2.2 and 2.3, equation (2.5), and noting that $z_{i} \sim G\left(p_{0}+p_{i}, \sigma_{i}, \mu_{i}+\mu_{0} \sigma_{i} / \sigma_{0}\right)$, yields the density function of $z_{M}$ conditional $z_{i}$ for $i=1, \ldots, M-1$

$$
\begin{aligned}
f\left(z_{M} \mid z_{1}, \ldots, z_{M-1}\right)= & \int_{y_{0}} \prod_{i=1}^{M-1} \frac{\Gamma\left(p_{0}+p_{i}\right) \sigma_{i}^{p_{0}}\left(y_{0}-\mu_{0}\right)^{p_{0}-1}}{\Gamma\left(p_{0}\right) \Gamma\left(p_{M}\right) \Gamma\left(p_{i}\right) \sigma_{0}^{p_{0}} \sigma_{M}^{p_{M}}} \\
& \frac{\left(z_{i}-y_{0} \sigma_{i} / \sigma_{0}-\mu_{i}\right)^{p_{i}-1}}{\left(z_{i}-\mu_{i}^{*}\right)^{p_{0}+p_{i}-1}} \exp \left(-\left(y_{0}-\mu_{0}\right) / \sigma_{0}\right) \\
& \left(z_{M}-y_{0} \sigma_{M} / \sigma_{0}-\mu_{M}\right)^{p_{M}-1} \exp \left(\left(z_{i}-\mu_{i}^{*}\right) / \sigma_{i}\right) \\
& \exp \left(-\left(z_{M}-y_{0} \sigma_{M} / \sigma_{0}-\mu_{M}\right) / \sigma_{M}\right) \\
& \exp \left[-\left(z_{i}-y_{0} \sigma_{i} / \sigma_{0}-\mu_{i}\right) / \sigma_{i}\right] d y_{0},
\end{aligned}
$$

where $\mu_{i}^{*}=\mu_{0} \sigma_{i} / \sigma_{0}+\mu_{i}$. 
Letting $E\left[U_{C} \mid x_{1}, x_{2}, \ldots, x_{M-1}\right]=E\left(\exp \left(\gamma z_{M}\right) \mid x_{1}, x_{2}, \ldots, x_{M-1}\right), x_{0}=$ $y_{0}-\mu_{0}$ and using propositions 2.3 and 2.2 yields

$$
\begin{aligned}
E\left(\exp \left(\gamma z_{M}\right) \mid x_{1}, \ldots, x_{M-1}\right)= & \prod_{i=1}^{M-1} \frac{\sigma_{i}^{p_{0}} \Gamma\left(p_{0}+p_{i}\right)}{\sigma_{0}^{p_{0}} \Gamma\left(p_{0}\right) \Gamma\left(p_{i}\right)} \frac{1}{\left(1-\gamma \sigma_{M}\right)^{p_{M}}} \\
& \int_{x_{0}} x_{0}^{p_{0}-1} \frac{\left(z_{i}-x_{0} \sigma_{i} / \sigma_{0}-\mu_{i}^{*}\right)^{p_{i}-1}}{\left(z_{i}-\mu_{i}^{*}\right)^{p_{0}+p_{i}-1}} \\
& \exp \left(x_{0} \gamma \sigma_{M} / \sigma_{0}+\gamma \mu_{M}^{*}\right) d x_{0}, \quad \text { B.3. }
\end{aligned}
$$

where $\mu_{M}^{*}=\mu_{0} \sigma_{M} / \sigma_{0}+\mu_{M}$.

3. (The asset specific pricing kernel) Finally, substituting equations (B.1) and (B.3) into equation (2.2) and considering Proposition 2.3 yields the asset specific pricing kernel. 


\section{References}

Black, F., and M. Scholes (1973): The Pricing of Options and Corporate Liabilities. Journal of Political Economy, 81, 637-654.

Brennan, M. (1979): The Pricing of Contingent Claims in Discrete time Models. Journal of Finance, 34, 53-68.

Buhlmann, H. (1980): An Economic Premium Principle. ASTIN Bulletin, $11,52-60$.

Camara, A. (2005): Option Prices Sustained by Risk-Preferences. Journal of Business, 78, 1683-1708.

Gerber, H., and E. Shiu (1994): Option Pricing By Esscher Transforms. Transactions of Society of Actuaries, 46, 99-140.

Griffiths, G. (1990): Rainfall Deficits: Distribution of Monthly Runs. Journal of Hydrology, 115, 219-229.

Heston, S. (1993): Invisible Parameters in Option Prices. Journal of Finance, 48, 933-947.

Johnson, H. (1987): Options on the Maximum of the Minimum of Several Assets. Journal of Financial and Quantitative Analysis, 22, 277-283.

Johnson, N. L., S. Kotz, and N. Balakrishnan (1994): Continuous Univariate Distributions (Vol. 1), Wiley.

Kotz, S., N. Balakrishnan, and N. L. Johnson (2000): Continuous Multivariate Distributions (Vol. 1), Wiley.

Margrabe, W. (1978): The Value of an Option to Exchange One Asset for Another. Journal of Finance, 33, 177-186. 
Mathai, A. M., and P. G. Moschopoulos (1991): On a Multivariate Gamma. Journal of Multivariate Analysis, 39, 135-153.

Mayhew, S. (1995): Implied Volatility. Financial Analysts Journal, Jul-Aug, 8-20

Poon, S., C. and Granger (2003): Forecasting Volatility in Financial Markets: a review. Journal of Economic Literature, 41, 478-539.

Savickas, R. (2005): Evidence on Delta Hedging and Implied Volatilities for the Black-Scholes, Gamma, and Weibull Option Pricing Models. Journal of Financial Research, 28, 299-317.

Schroder, M. (2004): Risk-neutral parameters shifts and derivatives pricing in discrete time. Journal of Finance, 59, 2375-2401.

Simpson, J. (1972): Use of Gamma Distribution in Single-Cloud Rainfall Analysis. Monthly Weather Review, 100, 309-312.

Stapleton, R. C., and M. G. Subrahmanyam (1984): The Valuation of Multivariate Contingent Claims in Discrete Time Models. Journal of Finance, $39,207-228$.

Stulz, R. (1982): Options on the Minimum or the Maximum of Two Risky Assets: Analysis and Applications. Journal of Financial Economics, 10, 161-185.

Vitiello, L., and S. Poon (2010): General equilibrium and preference free model for pricing options under transformed Gamma. Journal of Futures Markets, 30, 409-431.

Wang, S. (2002): A Universal Framework for Pricing Financial and Insurance Risks. ASTIN Bulletin, 32, 213-234. 
Wang, S. (2003): Equilibrium pricing transforms: new results of Buhlmann's 1980 economic model. ASTIN Bulletin, 33, 57-73.

Zhou, Z. (1998): An Equilibrium Analysis of Hedging with Liquidity Constraints, Speculation, and Government Price Subsidy in a Commodity Market. Journal of Finance, 53, 1705-1736. 
Figure 1: Implied volatilities: Values are obtained from simulated prices using equation (4.2) for different strikes prices, with $\mu_{0}=\mu_{2}=1, \sigma_{0}=\sigma_{1}=\sigma_{2}=0.5$, $p_{0}=1$ (solid line), $p_{0}=2$ (dotted line).

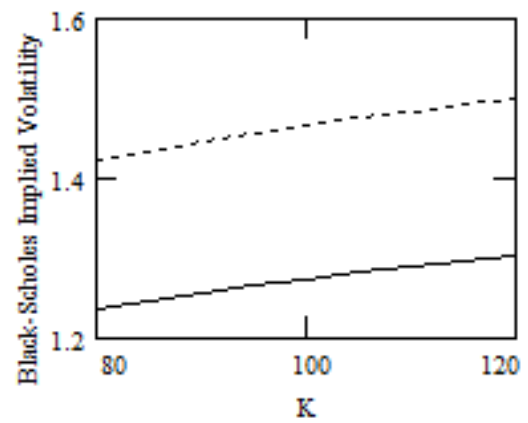

